\title{
Melt-Grafting of Maleimides Having Hindered Phenol Group onto Polypropylene
}

\author{
Taek Hyeon Kim and Namgun Lee \\ Department of Applied Chemistry and The Research Institute for Catalvis, \\ Chonnam Kational Lniversity, Gwangu 500-757, Korea \\ Recened July 19, 2003
}

\begin{abstract}
Monomeric antioxidant 1 was prepared by the reaction of 3.5-di-tert-butyl-4-hydroxybenzyl alcohol with $\mathrm{N}$ [4-(chlorocarbonyl)phenyl]maleimide in the presence of imidazole. Monomeric antioxidant 2 . bearing carbamate group, was synthesized from the reaction of 3.5-di-tert-butyl-4-hydroxybenzyl alcohol and azidomaleimide. Antioxidant 3 was prepared by the reaction of $\mathrm{N}$-(4-hydroxyphenyl)maleinide and 3-(3,5-ditert-butyl-4-lydroxyphenyl) propionic chloride in the presence of trietlylamine. These reactive antioxidants were grafted onto polypropylene (PP) by melt-processing with free radical initiators in a mini-max moulder. From the infrared spectra of the grafted PP. it was found that the monomeric antioxidants were grafted onto PP. IR spectroscopic methods were used for the quantitative determination of the extent of grafting of monomeric antioxidant. To optimize the reaction conditions. the influences of the concentration of DCP, monomeric antioxidant. reaction time and temperature on the extent of grafting were studied.
\end{abstract}

Key Words : Hindered phenol antioxidant. Melt grafting. Poly'mer-bound antioxidant

\section{Introduction}

Polypropylene. one of the most widely used polymers, is more susceptible to the oxidation than other polyolefins because of forming more stable alkyl radicals and need more antioxidants. Oxidation reactions are enlhanced at elevated temperatures during the processing of the polymer. To protect thermal oxidation of polymers. hindered phenol antioxidants with 2.6-di-fert-butylphenol group are widely used as effective antioxidants. ${ }^{1,2}$ Low molecular weight antioxidants, however, easily vaporize and have poor extraction resistance, shortening the service life of polymer. Physical loss of antioxidants constitutes a major concern in the reuse of recoverable plastics and the medical and food equipment for safety as well as in long-term use of polymers. Several approaches to more permanent antioxidant have been reported and among these the polyolefin-bound antioxidants, the functionalization of polyolefins with reactive antioxidants. provide the most attractive answer guaranteeing their performance in polymer matrix. ${ }^{3.6}$

Munteanu and Csunderlik reported the grafting of methacrylate, cinnamic acid, and styrene bearing the 2.6-di-tertbutylphenol group onto high density polyethylene using initiators. ${ }^{8}$ Al-Malaika and Suharty studied the melt grafting of acrylate onto PP in the presence of the trifunctional coagent trimethylol propane triacrylate using free radical initiator to give high grafting yields. ${ }^{\text {} b}$ However, acrylic monomers in grafting reactions are easy to homopolymerize. As a part of our program of polymer-bound additive. ${ }^{9}$ we report the melt graft polymerization of newly designed antioxidants $1-3$ bearing maleimide onto PP in a mini-max moulder to apply it industrially to the reactive extrusion.

Corresponding author. e-mail: thlim achonnam.ac.kr

\section{Experimental Section}

Chemicals and Equipment. The unstabilized PP powder was supplied by Samsung General Chemicals, Ltd with a melt flow index of $10.0 \mathrm{~g} / 10 \mathrm{~min}$ at $230^{\circ} \mathrm{C} / 2.16 \mathrm{~kg}$ and a density of $0.91 \mathrm{~g} / \mathrm{cm}^{3}$. Reagent grade dicumyl peroxide (DCP). and 2,2'-azobisisobutyronitrile (AIBN) were used without further purification whereas benzoyl peroxide (BPO) was purified by recrystalization in ether. The melt grafting reaction was carried out in a mixer. MINI-MAX MODEL CS 183MM-065. Custom Scientific Instruments Inc. ${ }^{10}$ IR spectra were recorded on Nicolet FT-IR.

General Grafting and Purification Procedure. All components. including the PP powder. purely prepared monomeric antioxidant 1-3 and initiator were fully mixed. The resulting dry powder mixture was charged into the chamber of mini-max moulder, which was processed at 60 $\mathrm{rpm}$ under nitrogen atmosphere. To get the optimal reaction conditions. a series of exploratory grafting reactions were performed by changing the initiators type (DCP. BPO. and AIBN) and concentrations $(0.3-2.0 \mathrm{mmol}$ per $100 \mathrm{~g}$ of PP), monomer concentrations $(0.05 .0 .20 \mathrm{mmol}$ per $100 \mathrm{~g}$ of PP), reaction time $(4-12 \mathrm{~min})$, and temperature $\left(160-200^{\circ} \mathrm{C}\right)$. It is known that grafting reaction includes some unavoidable homopolymerization. The reaction product was purified by dissolving in refluxing sylene, and precipitating in dichloromethane. The precipitated grafted PP was filtered, washed with more dichloromethane and dried in vacuum oven at 80 ${ }^{\circ} \mathrm{C}$. The ungrafted antioxidant, homopolymer, and peroxide were dissolved in the dichloromethane solution. while grafted and ungrafted PP was precipitated out. Successive reprecipitation showed that a one-time process was adequate to remove the homopolymer and unreacted monomer

Determination of Grafting Yield. The purified PP was analyzed by infrared (IR) spectroscopy using films compression 
moulded at $170^{\circ} \mathrm{C}$ and 2,000 psi for $5 \mathrm{~min}$. To determine the degree of grafting, the peak at $2720 \mathrm{~cm}^{-1}$ from the PP was chosen as a reference. ${ }^{7}$ A carbonyl peak at about $1720 \mathrm{~cm}^{-1}$ originated from the grafted antioxidant, was chosen as the target. The integral ratios of carbonyl peak $/ 2720 \mathrm{~cm}^{-1}$ were used as a convenient measure for the relative degree of grafting. The IR calibration curve for determining the grafting yield was established by mixing the $\mathrm{PP}$, monomeric antioxidants in different concentration, and DCP, recording the IR spectrum. The curve of monomer concentration versus integral ratio of carbonyl peak/2720 $\mathrm{cm}^{-1}$ was plotted assuming that the difference of absorbance between mixed and grafted monomer can be ignored. Grafting yield was calculated as follows:

$$
\text { Grafting yield }(\%)-\mathrm{M}_{\mathrm{i}} / \mathrm{M}_{\mathrm{i}} \times 100
$$

where $M_{t}$ and $M_{i}$ present the mass of the grafted monomer onto $\mathrm{PP}$ and initially used monomer, respectively.

Solvent Extraction and Thermal Oxidation. Fxtraction of films with dichloromethane was achieved in Soxhlet extractor for 3 days. Thermal oxidation of films before and after extraction was carried out in an oven at $120^{\circ} \mathrm{C}$ in air and monitored with FT IR spectroscopy using the peak height ratio of $1720 / 2720 \mathrm{~cm}^{-1}$ to assess the extent of oxidation of polymers, ${ }^{11.12}$ because the oxidation of PP generated the carbonyl peak at $1720 \mathrm{~cm}^{-1}$.

\section{Results and Discussion}

Synthesis of Monomeric Antioxidants. Monomeric antioxidants 1-3 were prepared as shown in Scheme 1 according to our previous procedure." Monomeric antioxidant 1 was prepared by the reaction of 3,5 -di-fert-butyl-4-hydroxybenzyl alcohol with $N$-[4-(chlorocarbonyl) phenyl]maleimide in the presence of imidazole in $60 \%$ yields. The stretching peak of the carbonyl group appeared at $1716 \mathrm{~cm}^{-1}$. Monomeric antioxidant 2, bearing carbamate group, was synthesized from the reaction of 3,5-di-tert-butyl-4-hydroxybenzyl alcohol and azidomaleimide in reflux in $95 \%$ yield. The stretching peak of the carbonyl group appeared at $1713 \mathrm{~cm}^{-1}$ Antioxidant 3 was prepared by the reaction of $\mathrm{N}$-(4hydroxyphenyl)maleimide and 3-(3,5-di-tert-butyl-4-hydroxyphenyl) propionic chloride in the presence of triethylamine in $60 \%$ yield, showing the strong stretching carbonyl bands in 1756 and $1718 \mathrm{~cm}^{-1}$.

Grafting Reaction and IR Spectra of Grafted PP. The most common method to bind the additives into PP chains by reactive extrusion involves free radical-induced grafting.

$$
\begin{aligned}
& \mathrm{RO}-\mathrm{OR} \stackrel{\Delta}{\longrightarrow} 2 \mathrm{RO} \text {. }
\end{aligned}
$$

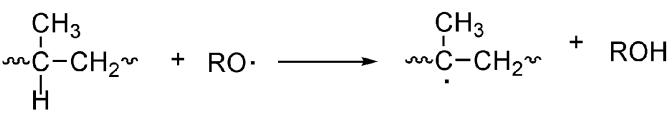

$$
\begin{aligned}
& \text { nomopolymer }
\end{aligned}
$$

Scheme 2. Outline of free radical indued gratting reaction and side reaction.<smiles>CC(C)(C)c1cc(CO)cc(C(C)(C)C)c1O</smiles><smiles>CCCCCCCCCOC(=O)c1ccc(N2C(=O)C=CC2=O)cc1</smiles><smiles>CC(C)(C)c1cc(CO)cc(C(C)(C)C)c1O</smiles><smiles>CC(C)(C)c1cc(COC(=O)Nc2ccc(N3C(=O)C=CC3=O)cc2)cc(C(C)(C)C)c1O</smiles><smiles>O=C1C=CC(=O)N1c1ccc(O)cc1</smiles><smiles>CC(C)(C)c1cc(CCC(=O)Cl)cc(C(C)(C)C)c1O</smiles>

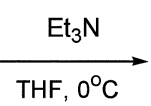<smiles>CC(=O)Oc1ccc(N2C(=O)C=CC2=O)cc1</smiles>

Scheme 1. Synthesis of monomeric antioxidants. 


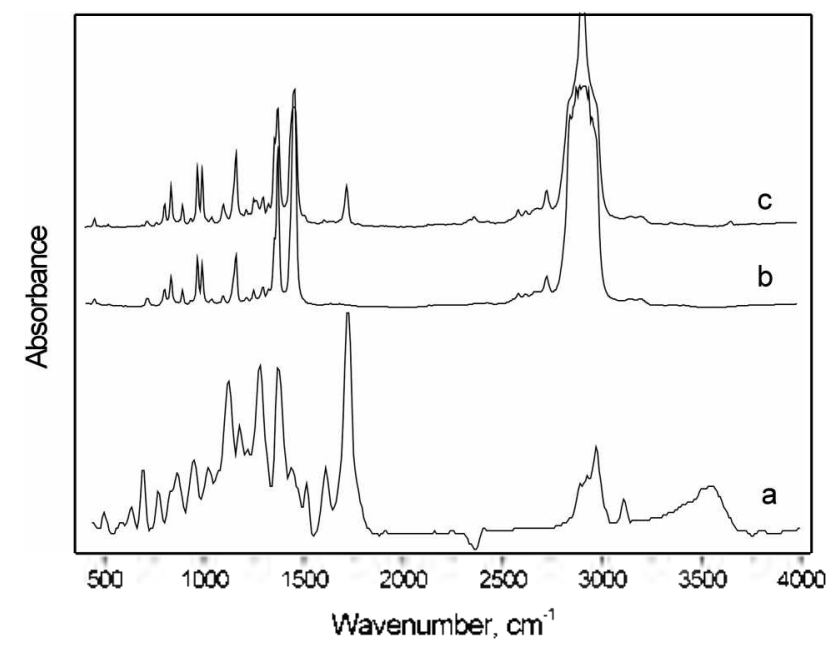

Figure 1. FT IR spectra, (a) the monomeric antioxidant 1, (b) the pure PP, (c) the grafted PP with 1.

Our designed monomers 1-3 bearing maleimide group could be used for the addition reaction to the alkyl radicals of PP (Schene 2). Hindered phenolic antioxidants are free radical scavengers to stabilize the radical in PP formed in processing. However. it is known that in general hindered phenolic antioxidants may not trap the alkyl radicals $\left(R^{\circ}\right)$ but the peroxy radicals ( $\mathrm{ROO}$ ) in the stabilization mechanism. ${ }^{13}$

Monomers 1-3 were grafted onto PP in the presence of free radicals. Grafting of 1-3 onto PP was confinned by IR spectroscopy. Figure 1-3 shows the infrared spectra of the monomeric antioxidants, the pure PP. and the grafted PP prepared under nitrogen. The spectra of the grafted PP show the bands around $1720 \mathrm{~cm}^{-1}$, which correspond the peak of the carbonyl group of the monomeric antioxidants 1-3. Also another peak due to $\mathrm{OH}$ group appears. During the grafting reaction, the band of $1720 \mathrm{~cm}^{-1}$ might also result from the degradation by the thermal oxidation of PP. To examine this side reaction. the grafting reaction was performed with 4 mmol of DCP per $100 \mathrm{~g}$ of PP for $10 \mathrm{~min}$ at $190^{\circ} \mathrm{C}$ without

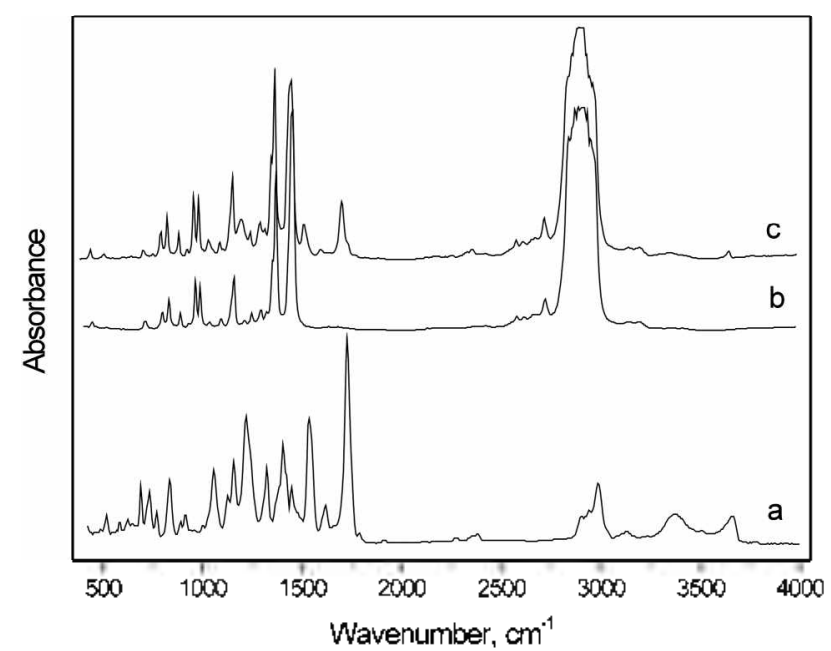

Figure 2. FT IR spectra, (a) the monomeric antioxidant 2, (b) the pure $P P,(c)$ the gratted $P P$ with 2.

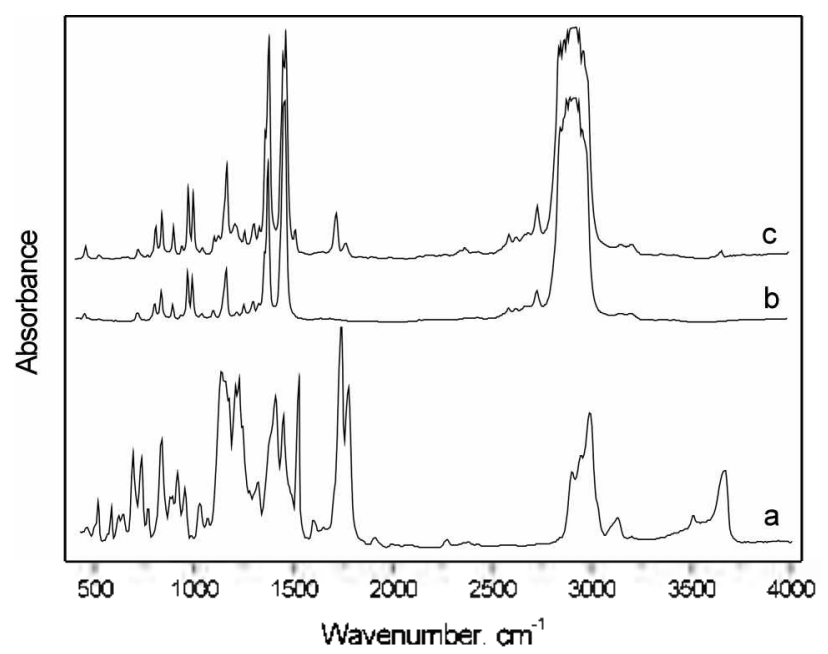

Figure 3. FT IR spectra, (a) the monomeric antioxidant 3, (b) the pure $P P$, (c) the gratted $P P$ with 3.

the monomer. The intensity of peak at $1720 \mathrm{~cm}^{-1}$ almost did not increase compared to the original PP. Thus. we confirmed that the peak at $1720 \mathrm{~cm}^{-1}$ after grafting reaction totally come from monomeric antioxidants. During the melt grafting in PP, three competing reactions could occur. such as the homopolymerization of monomer. the desired graft reaction, and $\beta$-scission (Scheme 2). Therefore, it was needed to investigate the effects of DCP concentration, monomer concentration, and reaction time and temperature to optimize the grafting reaction conditions blocking the side reactions.

Effect of Initiator Type and Concentration on Grafting Reaction. Three different initiators were tested, two peroxy initiators (DCP and $\mathrm{BPO}$ ) and one azo initiator (AIBN). The graft reaction was perfonmed with the monomer concentration of $5 \mathrm{mmol}$ per $100 \mathrm{~g} \mathrm{PP}$ and the initiator concentration of $2 \mathrm{mmol}$ per $100 \mathrm{~g}$ PP for $10 \mathrm{~min}$ at a reaction temperature of $180^{\circ} \mathrm{C}$. As can be seen in Figure 4 the extent of the grafting of monomers $\mathbf{1 - 3}$ with $\mathrm{PP}$ was in the order of DCP $>\mathrm{BPO}>\mathrm{AIBN}$. Thus DCP was chosen to give the best grafting yield. The grafting yield increased with

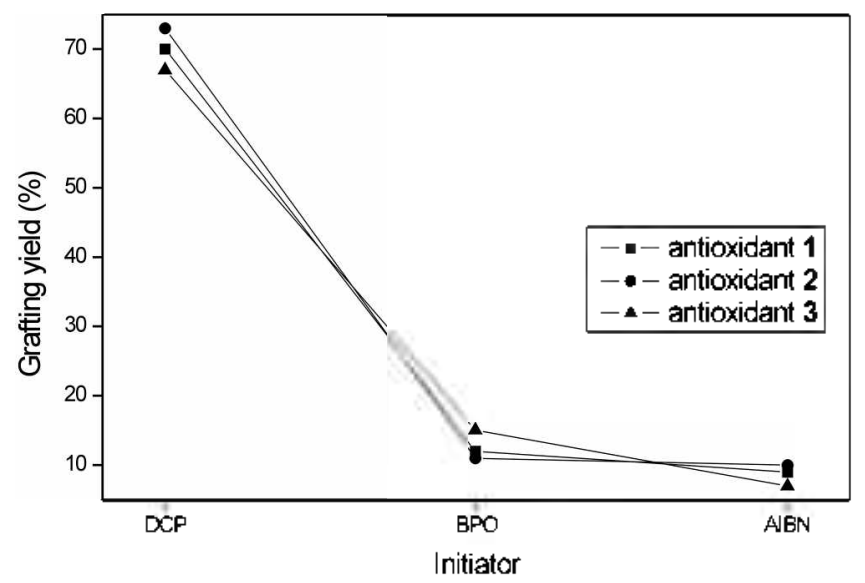

Figure 4. The influence of initiators on grafting yield (initiator concentration, $2 \mathrm{mmol}$ per $100 \mathrm{~g}$ of PP, monomer concentration, 10 mmol per $100 \mathrm{~g}$ of $\mathrm{PP}$; reaction temperature, $180^{\circ} \mathrm{C}$; time, $10 \mathrm{~min}$ ). 


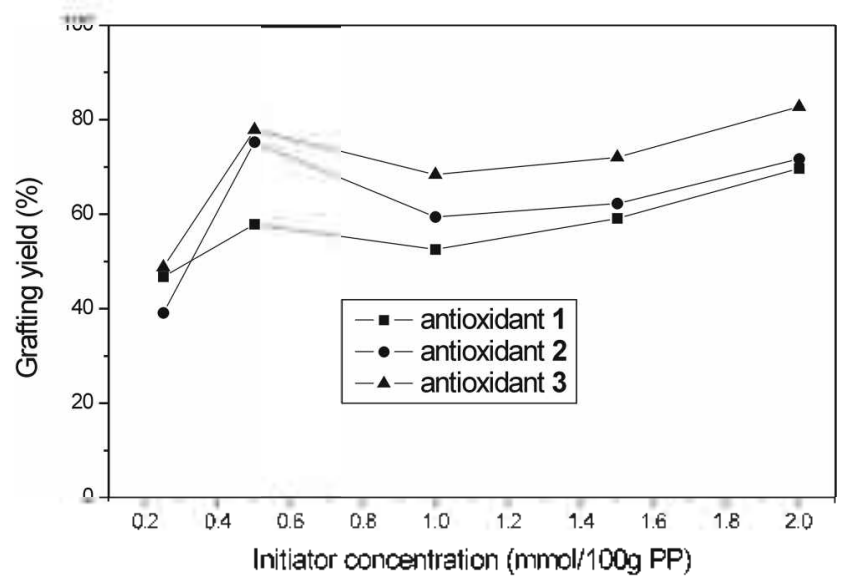

Figure 5. The influence of initiator DCP concentration on grafting yield (monomer concentration, $5 \mathrm{mmol}$ per $100 \mathrm{~g}$ of PP; reaction temperature, $190^{\circ} \mathrm{C}$ with $1,170^{\circ} \mathrm{C}$ with $2,180^{\circ} \mathrm{C}$ with 3 ; tine, 10 mini).

increasing half-life time $(t$,$) of the initiators. The t_{1: 2}$ values are as follows: ${ }^{14} \mathrm{AIBN} .7 .2 \mathrm{~min}$ at $100^{\circ} \mathrm{C}$. BPO, $19.8 \mathrm{~min}$ at $100^{\circ} \mathrm{C}$, and DCP, $1.7 \mathrm{hr}$ at $130^{\circ} \mathrm{C}$. The grafting reaction in the absence of initiator with the above reaction conditions did not occur. Thus thermally induced method did not give the grafting

The influence of a DCP concentration on the grafting yield is illustrated in Figure 5. For an each grafting reaction, the DCP concentration was varied over the range of $0.3-2.0$ mmol per $100 \mathrm{~g}$ of PP. With 1 the grafting were in the range of $45-65 \%$ yields depending on the initiator concentrations. With monomeric antioxidant 2 and 3. a rapid increase was observed at low initiator concentrations $(0.5 \mathrm{mmol})$. The grafting yield changed little with an increase in DCP concentration after an initial increase. Therefore, the concentration of DCP was optimized with $0.5 \mathrm{mmol}$ per $100 \mathrm{~g}$ of PP.

Effect of Reaction Time and Temperature. Figure 6 shows the influence of reaction time. The concentration of

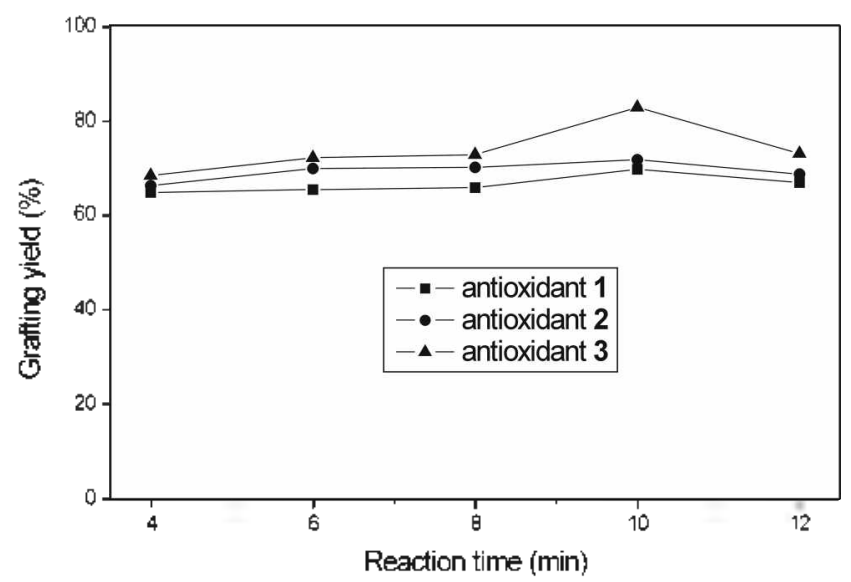

Figure 6. The influence of reaction time on grafting vield, DCP concentration ( $1 \mathrm{mmol}$ per $100 \mathrm{~g}$ of $\mathrm{PP}$; monomer concentration, 5 mmol per lo0 gof PP: reaction temperature, $190^{\circ} \mathrm{C}$ with $1,170^{\circ} \mathrm{C}$ with $2,180^{\circ} \mathrm{C}$ with 3

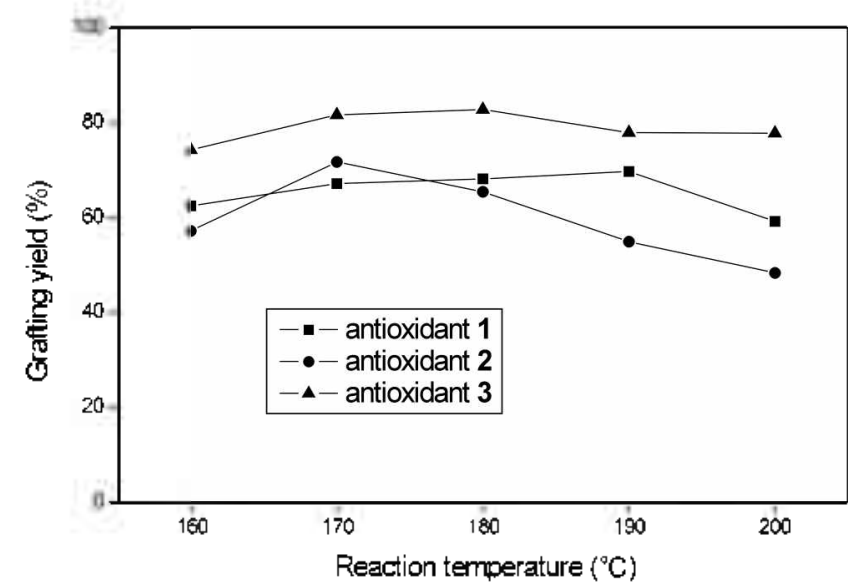

Figure 7 . The influence of reaction temperature on grafting yield (DCP concentration, $2 \mathrm{mmol}$ per $100 \mathrm{~g}$ of PP; monomer concentration, $10 \mathrm{mmol}$ per $100 \mathrm{~g} \mathrm{PP}$; time, $10 \mathrm{~min}$ ).

DCP and monomer is fixed at $1 \mathrm{mmol}$ and $5 \mathrm{mmol}$ per $100 \mathrm{~g}$ of $P P$, respectively. Grafting yield increased slightly with an increase in reaction time up to $10 \mathrm{~min}$. After $10 \mathrm{~min}$. the graft yield decreased. The decrease in the grafting yield after $10 \mathrm{~min}$ can be a result of degradation of formed grafts. ${ }^{15}$ The influence of temperature on grafting was also speculated (Figure 7 ). The best grafting was performed at $170^{\circ} \mathrm{C}$ with 1. $190^{\circ} \mathrm{C}$ with 2 , and $180^{\circ} \mathrm{C}$ with 3 . The grafting yield increased slightly to the optimal temperature and then decreased as temperature was further increased. The grafting yield decreases in the high temperature probably because faster chain termination at high temperature leads to short graft length. ${ }^{15}$

Effect of Monomer Concentration on Grafting Reaction. Figure 8 shows the effect of monomer concentration on grafting yield. The reaction was performed in a DCP concentration of $2 \mathrm{mmol}$ per $100 \mathrm{~g}$ of $\mathrm{PP}$ at a reaction temperature of $190^{\circ} \mathrm{C}$ with $1,170^{\circ} \mathrm{C}$ with 2 , and $180^{\circ} \mathrm{C}$ with 3 for $10 \mathrm{~min}$. Grafting yield decreased substantially as the monomer concentration increased. This behavior is

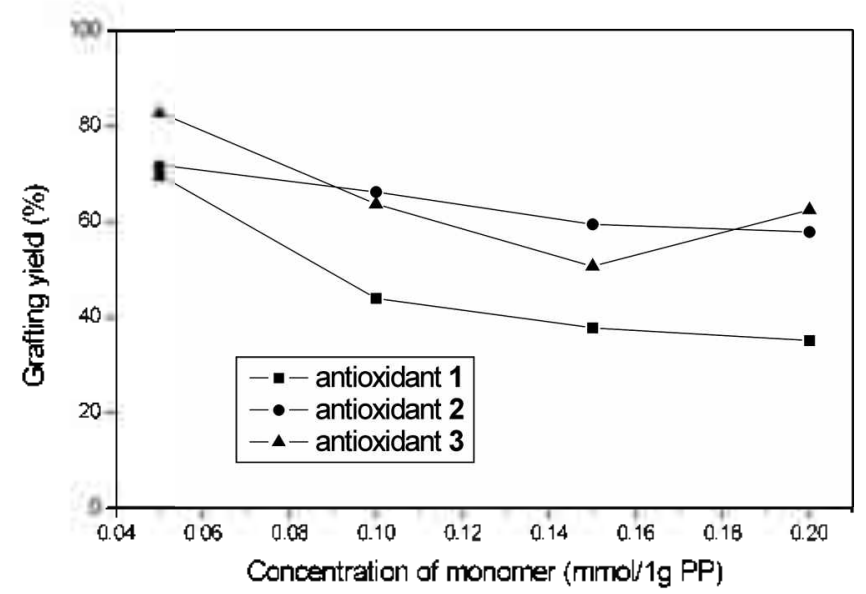

Figure 8. The influence of monomer concentration on grafting reaction (DCP concentration, $2 \mathrm{mmol}$ per $100 \mathrm{~g}$ of $\mathrm{PP}$; reaction temperature, $190^{\circ} \mathrm{C}$ with $1,170^{\circ} \mathrm{C}$ with $2,180^{\circ} \mathrm{C}$ with 3 : time, 10 min', 


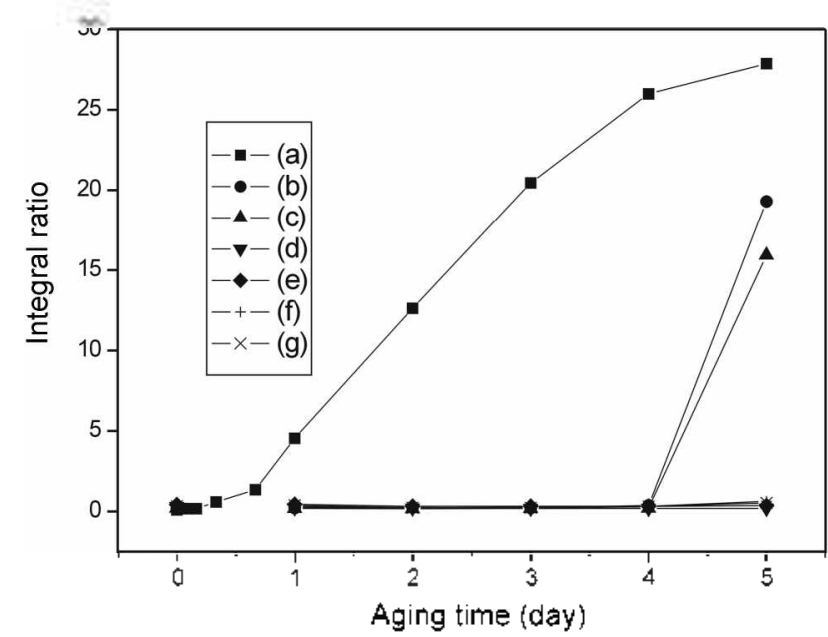

Figure 9. Antioxidative effect of the grafted PP before extraction, (a) the pure PP, (b) 0.2 wt $\%$ blended PP with 1 , (c) 0.2 wt \% blended PP with 2 , (d) 0.2 wt \% blended PP with 3 , (e) 0.2 wt \% Grafted PP with 1, (f) 0.2 wt \% Grafted PP with 2 , (g) 0.2 wt \% Grafted PP with 3 .

probably a result of the incompatibility between PP and monomer. The polar monomer molecules tend to form aggregate dispersed into the PP matrix. As the amount of monomer increased. the proportion of monomer located inside the phase did. Consequently the monomer became inaccessible to the free radical site of polymer. However the absolute amount of grafted mononer increased as monomer concentration increased.

Antioxidative Effect of Grafted PP Before and After Solvent Extraction. The change in peak height ratio of $1720 / 2720 \mathrm{~cm}^{-1}$ versus aging time is shown in Figure 9 (before the extraction) and 10 (after the extraction). The carbonyl content of unstabilized PP filnts drastically increased. while PP blended with the monomeric 1-3 and the PP-bound antioxidant films showed no increase of $1720 / 2720 \mathrm{~cm}^{-1}$ ratios for 4 days. However in 5 days monomers 1-2 began to degrade due to their evaporation but monomer 3 still blocked the polymers from thermal oxidation. After solvent extraction only the films blended with the monomeric 1-3 exhibited the increased peak of $1720 / 2720 \mathrm{~cm}^{-1}$ ratio because nonomeric antioxidants was lost. while the PP-bound antioxidant filnts showed no increase of $1720 / 2720 \mathrm{~cm}^{-1}$ ratios for 5 days. These prelininary oxidative results confirm that antioxidant moiety is chemically bound to the PP chains.

\section{Conclusion}

New polymer-bound antioxidants were successfully prepared by melt grafting a new monomeric naleinide 1-3 onto PP in the presence of a DCP initiator. Monomer 3 was better grafting reagent over monomer 1 and 2 in perfonnance and grafting yields. Also 3-(3.5-Di-tert-butyl-4-hydroxyphenyl)propionate group composed of monomer 3 was found to be more resistant to oxidation into quinone mojety than 3.5-ditert-butyl-4-hydroxyphenylnethyl alcohol group of 1 and 2.

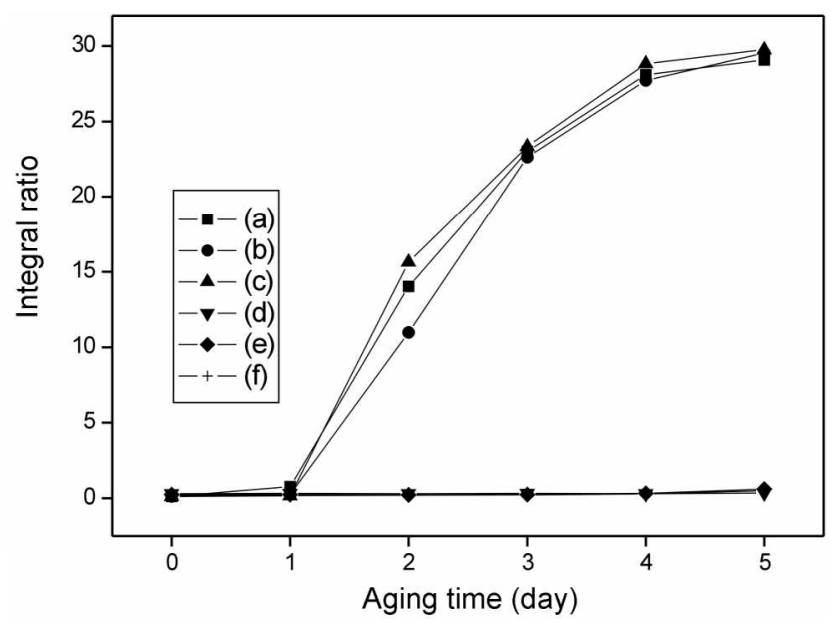

Figure 10. Antioxidative effect of the grafted $\mathrm{PP}$ after extraction, (a) 0.2 wt \% Blended PP with 1 , (b) 0.2 wt \% Blended PP with 2 . (c) 0.2 wt \% Blended PP with 3 , (d) 0.2 wt \% Gratted PP with 1, (e) 0.2 wt \% Grafted PP with 2, (f) 0.2 wt \% Grafted PP with 3 .

which gave lower yellowness. The presence of grafting was confirmed by the appearance of IR peak of the carbonyl of monomeric antioxidants in reacted PP. The grafted PP possessed some stabilizing effect against thermal oxidation with oven aging in air at $120^{\circ} \mathrm{C}$ for 5 days indicating no intensity change of IR peak at $1720 \mathrm{~cm}^{-1}$.

Acknowledgment. This work was supported by the grant from the Brain Korea 21 of the Ministry of Education. The support of the Korea Basic Science Institute (Grvangju branch) is also acknowledged.

\section{References}

1. Lutz. Jr, J. T. Themoplastic Polnter Additives; Theon and Practice, Marcel Dekker: New York, 1989.

2. Gugumus. F. Plastics Additives. 3rd Ed.: Ostord Univ. Press: New York. 1990

3. Al-Malaika. S. Chemtech. 1990. 366.

4. Munteanu. D. Developments in Polnter Stabilization-8, Scott. G.. Ed: Applied Science Publishers: London, 1987; Chapter 5.

5. Pospisil, J. Angew Maknol Chem. 1988. 158159, 221.

6. Kuczkowski. T. A.: Gillick. T. G. Rubber Chem. Techol. 1984. 57. 621 .

7. Vainic. T.: Hu. G.-H.: Lambla. M.: Seppala. J. V. J. Appl. Polym. Sci. $1996,61,843$.

8. (a) Munteanu. D.: Csunderlik, C. Polm. Deg. Stab. 1991. 31, 295 (b) Al-Malaika. S.: Suharty, N. Polym. Deg. Stab. 1995, $19,77$.

9. (a) Kim. T. H.: Kim. H.-K: Oh. D. R.: Lee. M. S.: Chae. K. H.: Kaan1g. S. J Appl. Polym Sci 2000. 77. 2968. (b) Oh. D. R.: Kiml. H.-K.: Lee. N.: Chae. K. H.: Kaang. S.: Lee. M. S.: Kim. I. H. Bull. Sorean Chem. Soc. $2001,22,629$.

10. Marechal. P. Chiba. T.: Inoue, T. Pohnt Networks Blends 1997. 7.61.

11. Pan. T. Q.: Lau. W. W. Y. Polym Degrad. Stab. 1996. 53.153.

12. He. M. B. Polym. Degrad. Stab. 1991. 33. 3367.

13. Matsumoto. A.: Yamagishi. K.: Aoki. S. f. Polvm Sci. Part A: Polm. Chen 1994. 32,917.

14. Odian. G. Principles of Polnterization; Wiley: New York. London. Sydney: Toronto. 1991; p 215

15. Song. Z: Baker. W. E. Angew Hahomol Chem 1990. 181. 1. 